Revista Electrónica Complutense de Investigación en Educación Musical ISSNe: 1698-7454

http://dx.doi.org/10.5209/RECIEM.59378

\title{
Investigación-creación en los trabajos de grado de pregrado en música. Un estudio de caso
}

\author{
Blanca Luz Morales Ortiz; ; Ana Margarita Giraldo Veloza²; Yamira Rodríguez Núñez; \\ Patricia Casas Fernández ${ }^{4}$
}

Recibido: 4 de marzo de 2018/ Aceptado: 8 de julio de 2018

Resumen. En Colombia, gracias al principio de Autonomía Universitaria consagrado en el artículo 69 de la Constitución Nacional de 1991, las universidades establecen sus titulaciones, programas y asignaturas contemplando las disposiciones previstas por el Ministerio de Educación Nacional. Uno de los aspectos a establecer dentro de esta autonomía son los requisitos de grado. En el reglamento estudiantil del año 2003 de la Universidad El Bosque, se incluye como requisito que es necesario cursar el $100 \%$ de los créditos del programa o mínimo el 50\%, en el caso que el estudiante sea de transferencia, y que adicionalmente se debe presentar un trabajo de grado, el cual es determinado por el Consejo de Facultad que rige al programa cursado por el estudiante. En el caso del programa de Formación Musical, dichos trabajos fueron realizados desde el año 2004 hasta el 2014 en el espacio académico denominado "Proyecto Artístico". El objetivo de la presente investigación es establecer en qué consistieron los proyectos desarrollados en la modalidad de investigación-creación realizados en dicha década, contemplando los productos creativos en los diferentes énfasis que ofrece la carrera: Arreglos Musicales, Ejecución Instrumental, Composición, Enseñanza Instrumental e Ingeniería de Sonido. El estudio es bibliográfico, descriptivo y de corte mixto, en el que se tuvo como principal técnica de recolección de datos la elaboración de fichas de Resúmenes Analíticos Especializados (RAE). Como conclusiones se pudo determinar que los trabajos de investigación-creación corresponden a tres diseños: Bucle, Ortodoxo e Invertido y que los Énfasis con mayor producción vinculada a este tipo de investigación son Arreglos Musicales, Composición y Ejecución Instrumental.

Palabras clave: investigación-creación; trabajo de grado; pregrado en música; formación musical.

\section{[en] Research-creation in the Works of undergraduate degree in music. A case study}

Abstract. In Colombia, thanks to the principle of University Autonomy enshrined in Article 69 of the National Constitution of 1991, universities establish their degrees, programs and subjects, contemplating the provisions envisaged by the Ministry of National Education. One of the aspects to establish within this autonomy are the degree requirements. In the student regulation of the year 2003 of the University El Bosque, it is mandatory to present a project or thesis determined by the Faculty Council and accomplish $100 \%$ of the

1 Universidad El Bosque

moralesblanca@unbosque.edu.co

2 Universidad El Bosque

giraldoana@unbosque.edu.co

3 Universidad Del Atlántico

yamirarodriguez@mail.uniatlantico.edu.co

$4 \quad$ Universidad Industrial de Santander

pcasas@uis.edu.co 
credits of the program, or at least 50\% in the case of transfer students. In the Music Degree Programs, these works were carried out from 2004 to 2014 in the academic area called "Artistic Project". The aim of the present research is to establish the purpose and development of the research-creation projects modality, carried out in that decade, contemplating the creative products in the different emphases offered by the career: Musical Arrangements, Instrumental Performance, Composition, Instrumental Pedagogy and Sound Engineering. The study is a bibliographical, descriptive and mixed-cutting study, in which the elaboration of Specialized Analytical Summaries (SARs) was used as the main technique of data collection. As conclusions it was determined that the research-creation works correspond to three designs: Loop, Orthodox and Inverted and that the Emphasis with greater production related to this type of investigation are Musical Arrangement, Composition and Instrumental Performance.

Key words: research-creation; work of degree; undergraduate in music; musical formation.

Sumario: 1. Introducción. 2. Materiales y métodos. 3. Resultados 4. Discusión. 5. Conclusiones e implicaciones. 6. Referencias bibliográficas.

Cómo citar: Morales, B. L.; Giraldo, A. M., Rodríguez, Y.; Casas P. (2018). Investigación-creación en los trabajos de grado de pregrado en música. Un estudio de caso, en Revista Electrónica Complutense de Investigación en Educación Musical, 15, 97-118.

\section{Introducción}

Cada uno de los procesos que generan diferentes resultados en las artes están enmarcados por el ejercicio natural que demanda cualquier creación: el desarrollo de una o varias ideas que parten de un medio material, sonoro o visual, lo cual implica una conciencia de los procedimientos cuyo origen no necesariamente posee un carácter intelectual puro, pero que responden de manera diversa a la necesidad de comunicación y exteriorización de lo emotivo e imaginativo, por medio de un lenguaje concreto o abstracto.

Para Daza (2009) durante las últimas décadas se ha volcado un serio interés en considerar la Investigación-Creación como una forma de metodología que permite el estudio formal de dichos procesos, para ampliar y enriquecer las diferentes formas de abordar la investigación, nutrir los campos del conocimiento y llevarlos a la materialización de la creación y el arte.

La Investigación-Creación es considerada una manera a través de la cual el campo de las artes busca posicionar los procesos creativos para estar al nivel de la comunidad académica científica en debates sobre la generación y producción de conocimiento, consolidar una comunidad académica artística para las artes y tomar prestados métodos de investigación de las Ciencias Sociales, con el fin de desarrollar un método investigativo propio y validar prácticas artísticas dentro del campo académico (Daza, 2009).

Según Daza (2009) para poder comprender en qué consiste la InvestigaciónCreación es necesario ahondar y analizar lo que sucede con la creación en el arte, desde los tres elementos que han estado presentes en ella: el sujeto creador o artista, el objeto o práctica artística y el espectador o público receptor de la propuesta artística. Este mismo autor afirma que "no hay obra sin creador, no hay obra sin espectador, pero no hay creador sin obra" (Daza, 2009, p. 87).

En este punto se hace referencia exclusivamente a las propuestas con componentes de interactividad, creaciones electrónicas o propuestas digitales interactivas, en 
las que el espectador es quien elabora, termina, complementa, y vivencia la obra. Ya no es algo para ver y contemplar, las propuestas artísticas presentan momentos, ambientes, eventos, y vivencias tal vez imposibles en la realidad, tal vez nuevas realidades que permiten nuevas experiencias que activan la presencia de quien está enfrente (Daza, 2009).

Scanner (2009) resalta que la Investigación-Creación incluye la reflexión escrita que acompaña el trabajo de creación, puesto que al existir un registro, ofrece un aprendizaje mixto y permite manifestar lo que permanece contenido en la expresión misma de la creación artística, como lo es la teoría enlazada y aplicada a una práctica. Esto no necesariamente responde a un rigor investigativo, pues la metodología se desarrolla desde la concepción y práctica artística en el proceder de la creación como metodología misma, sujeta a la particularidad del creador.

María José Contreras (2013) en su artículo titulado "la práctica como investigación: nuevas metodologías para la academia latinoamericana" complementa esta idea señalando lo siguiente:

Lo que define una práctica como investigación es que las preguntas o motivaciones iniciales solo puedan ser contestadas mediante la práctica. Esto implica que, si bien los aspectos conceptuales y la reflexión crítica están presentes, lo crucial sigue siendo la práctica. (p. 76)

Asimismo, Scanner (2009) resalta la relevancia de la Investigación - Creación dentro de la formación artística como generadora de conocimiento, ya que al combinar la práctica y su teoría permite precisar metodologías donde se identifican problemáticas claras, se plantean hipótesis de partida, se experimenta y extraen conclusiones:

Puede basarse en la práctica. En este caso, el estudiante se concentrará en una problemática específica en la que profundizará, y paralelamente realizará una labor de creación y exploración: redacción de una obra y reflexión sobre el proceso (...) La segunda opción es que esta reflexión se haga en la práctica. A partir de esta práctica, el estudiante comprueba en el laboratorio el fundamento de su hipótesis. (p. 324)

La Investigación-Creación es una indagación en la que se soluciona una situación problemática a partir de una experiencia creativa y que generalmente se relaciona con dos tipos de productos: la obra de arte y un texto de carácter reflexivo sobre la experiencia artística realizada con relación a la situación problema. En la investigación creación "se asumen los productos artísticos como objetos cognitivos." (Casti1lo, 2013, p. 57, citado en COLCIENCIAS, 2015, p. 126).

De forma similar, Eisner (1995) expresa que la Investigación-Creación como una evolución de los procesos de enseñanza de las artes "el aprendizaje artístico aborda el desarrollo de las capacidades para crear formas artísticas, para la percepción estética y para comprender el arte como fenómeno cultural" (p. 283).

En la investigación artística se busca ampliar y redefinir el concepto de investigación, precisar mejor los vínculos entre la investigación y la práctica, encontrar una 
finalidad que se centre en la creación y permita comprender las etapas y formas de dichos procesos de creación, validar la palabra del artista dentro de la construcción global del conocimiento enmarcado por un saber práctico, informado y argumentado desde su propia experiencia (Scanner, 2009). El autor define la práctica y finalidad ligadas dentro de la concepción y definición mismas de la Investigación - Creación de la siguiente manera:

Sus objetivos, métodos y finalidad modifican nuestra mirada sobre la investigación y su papel. Tiene por objetivo subvertir la investigación tal y como suele efectuarse, y hasta modificar nuestra visión de lo que debe ser la investigación para concebirla de otra forma. Quiere recuperar el poder para restituírselo al artista. Reivindica su derecho a contribuir al saber, a todo el saber, práctico y no práctico, con la misma legitimidad que la investigación más clásica. Se esfuerza por hacer cohabitar así, sin división, práctica y discurso, por convertir la práctica en un modo de investigación. (Scanner, 2009, p. 324)

Como se infiere, la Investigación-Creación o investigación artística tiene como meta llevar al lector a un conocimiento más profundo o concreto sobre determinados temas asociados a las artes. Según afirman López y San Cristóbal en su libro "Investigación artística en música", uno de los mayores retos de esta es "convertir algunas de las prácticas artísticas habituales en estrategias metodológicas formalizadas de investigación" (López-Cano y San Cristóbal, 2014, p. 126).

Por otro lado, Contreras (2013) hace énfasis en que la Investigación-Creación busca resistir el discurso verbal en la academia para generar conocimientos de carácter no verbal, dado que la obra artística no es un objeto de estudio, sino un agente capaz de generar conocimientos que van más allá del lenguaje verbal y las codificaciones matemáticas que tanto ha privilegiado la ciencia moderna. De otra parte, Haseman (2006) profundiza en esta característica de la investigación artística y afirma:

[Que] se expresa en formas no numéricas y simbólicas que van más allá de las palabras y los textos discursivos. Estas formas incluyen formas materiales de prácticas, de imágenes fijas o en movimiento, música o sonido, acción en vivo o códigos digitales. (p. 6)

Para López-Cano y San Cristóbal (2014) la Investigación-Creación en artes se diferencia de otros tipos de investigación en cuanto a que presenta diferentes características y procedimientos con relación a la recolección de datos. Con esto se hace referencia desde la práctica Musical a un método de recolección de datos en el cual el artista investiga, analiza, crea, compone y experimenta, "el rasgo que mejor caracteriza la investigación artística y la distingue de otro tipo de pesquisa académica, es que en ella la práctica Musical tiene un papel fundamental, ya sea a nivel técnico, interpretativo, creativo, escénico o artístico" (López-Cano y San Cristóbal, 2014, p. 123).

Siguiendo a López-Cano y San Cristóbal (2014), estos comentan que las características de la investigación en música se encuentran bajo diferentes ópticas en el 
desarrollo de conocimientos y mencionan dos aspectos que identifican la investigación en música: la investigación para la práctica artística y la investigación a través de esta:

La investigación para la práctica artística produce conocimiento o herramientas para el desarrollo de la actividad Musical en el más amplio sentido, como recursos teóricos y tecnológicos para la creación, interpretación, escucha y estudio de la música. La investigación a través de la práctica artística o investigación artística propiamente como tal, constituye un campo emergente de difícil definición. En general, aborda preguntas y problemas que no pueden ser atendidos en contextos carentes de un nivel de práctica artística alto o sin la participación de profesionales artísticos. Son indagaciones sobre problemas que atañen a la creación artística, que preocupan a la comunidad de creadores y que requieren de su particular experiencia y conocimiento para ser planteados y resueltos. (López-Cano y San Cristóbal, 2014, pp. 40-41)

Para estos autores, las funciones de la práctica Musical en la Investigación-Creación son variadas, pueden ser informativas, reflexivas, experimentales o vehiculares:

- Es reflexiva cuando se convierte en un lugar para pensar, encontrar similitudes y contradicciones, generar ideas o producir conceptualizaciones y soluciones.

- Es informativa cuando provee de información a la investigación.

- Es experimental cuando se convierte en espacio para comprobar, testear y evaluar diferentes soluciones o ideas o se convierte en el laboratorio de exploración artística y/o intelectual.

- Es vehicular cuando se convierte en recurso de comunicación de los resultados de la investigación. (López-Cano y San Cristóbal, 2014, pp. 126-127)

Teniendo en cuenta los aportes de los autores anteriormente presentados, la Investigación-Creación constituye un tipo de metodología investigativa que aún está en proceso de desarrollo. En el presente estudio denominado "Investigación-Creación en los trabajos de grado de pregrado en música. Un estudio de caso", se pretende establecer en qué consistieron los proyectos Artísticos del Programa de Formación Musical de la Universiad El Bosque, desarrollados en la modalidad de InvestigaciónCreación durante los años 2004-2014, y cuáles fueron los productos creativos de los diferentes énfasis que ofrece esta carrera.

La Universidad El Bosque es una institución privada sin ánimo de lucro, ubicada en la ciudad de Bogotá. Cuenta con Acreditación Institucional de Alta Calidad hasta el año 2020 otorgada por el Ministerio de Educación Nacional y tiene como compromiso el "crear una comunidad educativa integral, que articula las dimensiones biológicas, psicológicas y sociales de la persona ligada a su contexto" (Universidad El Bosque, s.f., párr. 1). Dentro de las Facultades que la constituyen, se encuentra la de Creación y Comunicación que abarca seis programas: Arquitectura, Arte Dramático, Artes Plásticas, Formación Musical, Diseño de Comunicación y Diseño Industrial.

Desde el programa de Formación musical se imparte la música "como fenómeno artístico, histórico, social y económico" (Universidad El Bosque, s.f., párr. 4), 
a partir de cinco énfasis: Composición, Arreglos Musicales, Ejecución, Enseñanza Instrumental y Producción.

Los proyectos artísticos del programa de Formación Musical se realizan desde el año 2004 y constituyen un requisito de grado, en donde se evidencian los aprendizajes adquiridos en la carrera. En el informe sobre el área de investigación realizado por su coordinadora en el año 2011, la Maestra Luz Ángela Gómez, se plantea que el Proyecto Artístico consiste en el desarrollo de una propuesta artística, "resultado de un proceso que corresponde a la formulación apropiada de un problema de conocimiento que induzca a la investigación, convirtiéndose en un producto de carácter creativo, relacionado con el énfasis al cual está inscrito el estudiante" (Gómez, 2011, p. 4).

Debido a que se desconocía el cómo se concebían estos trabajos desde el inicio del programa en el año 2004, ya que no existe documentación sobre este aspecto, y teniendo en cuenta que en la base de datos de la biblioteca de la Universidad no aparecen reseñados algunos trabajos realizados, se hizo necesario desarrollar un estudio sobre los proyectos artísticos, sus componentes, temáticas, líneas de investigación, manejo de fuentes bibliográficas, metodologías de investigación más empleadas, entre otros aspectos; con el fin de caracterizar los proyectos artísticos del programa. Dicho estudio se denominó: "Estado del arte de los Proyectos artísticos del programa de Formación Musical 2004-2014. Análisis comparativo desde sus diferentes énfasis", y se desarrolló durante los años 2015 y 2016, bajo el patrocinio de la Universidad El Bosque.

En la investigación se pudo establecer que los proyectos artísticos desde sus inicios fueron direccionados hacia el desarrollo de la investigación formativa. En las Políticas de Formación para la Investigación, Creación, Desarrollo e Innovación de la Universidad El Bosque (2015), se define la investigación formativa "como parte de la acción de enseñanza del docente, que tiene un fin pedagógico y corresponde al currículo" (pp. 17-18). Para que esta se desarrolle de una manera pertinente, debe ir acompañada de un proceso de Formación para la Investigación, en donde el estudiante obtiene las herramientas que le permiten desarrollar su trabajo de grado desde la óptica de la investigación. Ambas deben desarrollarse de manera conjunta, en tanto que en la Formación para la Investigación, se adquieren las herramientas investigativas y en la investigación formativa estas se llevan a la práctica (Universidad El Bosque, 2015).

La investigación formativa juega un papel importante en el proceso de aprendizaje a través de actividades propias de la investigación. En esta no se pretende obtener resultados científicos, comprendidos bajo conceptos estadísticos, sino que tiene como fin la construcción y organización de un conocimiento previo.

En este tipo de investigación se parte de un problema, el estudiante indaga, busca, revisa, relaciona y recolecta datos, generando así el aprendizaje de conocimiento. Recibe dicho nombre porque al ordenar y construir conocimiento sigue los pasos de la investigación en sentido estricto, siendo su producto de importancia local, sin necesidad de ser validado frente a una comunidad académica internacional. Los asesores y evaluadores que participan de la investigación no llevan a juicio inflexible de expertos los procesos y resultados, si no que promueven un espacio de experimentación donde uno de los fines, es inducir al estudiante en el gozo de saber más, sin perder la rigurosidad con que se haya planteado la meta (Hernández, 2003).

La investigación formativa cumple una función pedagógica que busca adentrarse en la investigación por medio de su práctica, impulsando a que los estudiantes logren 
comprender y adelantar una investigación científica, siendo esta una estrategia de aprendizaje mediante el descubrimiento y la construcción. (Morales, et al, 2018, p. 22). El Consejo Nacional de Acreditación (CNA) hace referencia a la investigación formativa como "una primera e ineludible manifestación de existencia de la cultura de la investigación en las instituciones de educación superior” (Restrepo, 2008, p. 5). Restrepo (2003) afirma:

El tema de la denominada investigación formativa en la educación superior es un tema-problema pedagógico que aborda, en efecto, el problema de la relación docencia-investigación o el papel que puede cumplir la investigación en el aprendizaje de la misma investigación y del conocimiento. (p. 18)

De esta manera, se justifica que la manera más objetiva de abordar el tema es desde el campo de la enseñanza - específicamente desde dos de las que para él son las principales estrategias del mismo- exponiéndolas de la siguiente manera: "Ellas son la estrategia de enseñanza expositiva o por recepción, más centrada en el docente y en el contenido, y la estrategia de aprendizaje por descubrimiento y construcción del conocimiento, más centrada en el estudiante" (Restrepo, 2003, p. 19).

Erquicia (2011) y Restrepo (2003), coinciden en afirmar que "parte de la problemática en la educación actual, es que la docencia universitaria se ha desarrollado con base en un modelo didáctico de corte transmisionista que ha dicotomizado lo profesional y lo investigativo" (p. 81). Del mismo modo hay similitudes encontradas con lo estudiado por González (s.f.) de la Universidad Sergio Arboleda, quien indica que "observamos el papel de la interdisciplinariedad en investigación y finalmente observamos cómo, a partir de la investigación formativa y la investigación en el aula, se promueven transformaciones en las prácticas educativas y en las construcciones curriculares" (párr. 3).

De otro lado Sandoval (2002) trae a colación los aportes de Robert Yin (1984) que en un texto publicado en (1985) nos acerca al estudio de caso, como una indagación empírica formativa que investiga un fenómeno contemporáneo dentro de su contexto real de existencia, cuando los límites entre el fenómeno y el contexto no son claramente evidentes y en los cuales existen múltiples fuentes de evidencia que pueden usarse; este puede incluir tanto estudios de un solo caso como de múltiples casos, donde se encuentran lineamentos generales para dar cuenta de las modalidades de muestreo no probabilístico que conducen a realizar observaciones o búsquedas selectivas en casos específicos, que son los referentes centrales de este artículo.

La investigación formativa involucra tanto al docente como al estudiante, teniendo como principal objetivo el generar un conocimiento por medio de la investigación; adicional a esto, también busca desarrollar destrezas investigativas con el fin de formar profesionales más íntegros. La Universidad El Bosque plantea los siguientes objetivos de la investigación formativa a partir de la definición aportada por Restrepo (2003) en el documento "Conceptos y aplicaciones de la investigación formativa y criterios para evaluar la investigación científica en sentido estricto" (p.18):

- Enseñar a investigar a docentes y estudiantes.

- Desarrollar habilidades cognoscitivas como la analítica, el pensamiento productivo y la solución a problemas. 
- Familiarizar a los estudiantes con las etapas de la investigación y los problemas que éstas plantean.

- Construir en los docentes la cultura de la evaluación permanente de su práctica.

Estudiante, docente y objeto de la investigación, son los tres componentes determinantes en la investigación formativa. Es deber de los dos primeros encontrarse en sincronía con el fin de abordar de manera adecuada el objeto de investigación. Cabe resaltar que la Universidad El Bosque basa los cimientos de sus componentes de investigación formativa en la perspectiva de Bernardo Restrepo (2003), quien afirma:

Para empezar, los trabajos de grado son oportunidad clara para hacer investigación formativa, cuando el estudiante cuenta con la fortuna de un asesor exigente y riguroso que cumple en verdad la función de asesorar la investigación, esto es, cuando hace comentarios sesudos, cuando obliga a rehacer partes, a refinar la conceptualización y la argumentación, cuando orienta la comparación (...) Pero no hay que esperar a que el estudiante esté a punto de culminar sus estudios de pregrado para inducirlo en esta práctica. (p. 17)

En la investigación del 2015 también se pudo establecer que todos los trabajos de grado estuvieron vinculados a las líneas de investigación de la Carrera de Formación Musical. Escorcia (2010) define "línea de investigación" como proyectos desarrollados sobre una misma temática, que parten de la iniciativa personal de un profesor que profundiza en su campo de conocimiento y/o de trabajos dirigidos por él, y que, a través del desarrollo y culminación de los mismos, convierten al docente en un especialista en el tema. "A medida que incursiona en la temática empieza a vérsele como un especialista rodeado de un contingente de alumnos interesados en desarrollar proyectos sobre el mismo tema"(Escorcia, 2010,p. 25). De esta manera una línea de investigación está compuesta por varios proyectos que brindan conocimientos y posibilitan el desarrollo de posteriores investigaciones en el mismo campo del conocimiento.

En el texto "Lineamientos y política para el fomento de la investigación y documentación en artes y música" del Ministerio de Cultura de Colombia, se mencionan seis líneas de investigación en el área de música: "Pedagogía", que trata temáticas referentes a las diferentes artes y sus didácticas; "Historia", "Culturas tradicionales, folklore y etnias", "Estética", orientada a las artes plásticas y lo filosófico; "Reflexión artística disciplinar o interdisciplinar", en la que se analizan las artes, sus áreas y las relaciones entre ellas; y "Tecnología", donde se tratan las nuevas tecnologías y su incidencia en las artes con respecto a los procesos creativos y las tecnologías para el arte (Ministerio de Cultura, s.f.).

Para el año 2015, cuando se desarrolla el estudio, la Facultad de Artes de la Universidad El Bosque, en donde se encontraba vinculada la carrera de Formación musical, contaba con tres líneas de investigación formuladas: Educación y Arte, Investigación Creación y Teoría estética y Arte; además de otras tres líneas pendientes por formular: Gestión, Desarrollo y producción; Salud, ciencia, tecnología y artes, y Subjetividad, desarrollo y espacios sociales (Cabanzo, 2015). Como se indicó anteriormente, al finalizar la investigación del año 2015 se pudo establecer que todos los trabajos de grado del periodo 2004 al 2014 estaban vinculados a las líneas de investigación existentes. 
La Investigación-Creación fue una de las líneas de investigación del programa de Formación Musical desde el año 2004 al 2014, y tuvo como objetivo central el incentivar la creación artística mediante proyectos investigativos y de creación, vinculados a la investigación formativa. (Cabanzo, 2015). En la última década la Investigación-creación ha sido uno de los temas a discutir en la investigación en artes, en donde se ha pensado hasta qué punto esta línea podría constituir o no, un modelo de investigación particular en las artes.

\section{Materiales y métodos}

El estudio es bibliográfico, descriptivo y de corte mixto. Según García (2004) los estudios descriptivos son "observacionales, en los cuales no se interviene o manipula el factor de estudio, es decir se observa lo que ocurre con el fenómeno en estudio en condiciones naturales, en la realidad" (p. 1). Para Hernández, Fernández y Baptista (2010) los estudios descriptivos "buscan especificar las propiedades, características y los perfiles de personas, grupos, comunidades o cualquier otro fenómeno que sea sometido a análisis" (p. 80).

Los estudios bibliográficos parten del análisis de documentos. Este trabajo corresponde a un estudio bibliográfico, ya que se analiza la totalidad de los 279 trabajos de grado del Programa de Formación Musical, correspondientes al periodo 2004-2014. Dichos trabajos fueron leidos a cabalidad y a partir del análisis de los diferentes apartados de cada proyecto, se desarrolló una ficha de Resumen Analítico Especializado (RAE).

El enfoque mixto aplica en esta investigación, dado que los datos obtenidos se analizan desde una perspectiva cuantitativa y otra cualitativa, buscando responder al planteamiento del trabajo. Luego de la primera fase, en la que se realizan las fichas RAE, la información es analizada utilizando SPSS con lo cual es posible establecer las frecuencias de las variables analizadas.

La técnica de recolección de datos empleada en esta investigación es una ficha de Resumen Analítico Especializado (RAE). A partir de las fichas RAE se pretende condensar la información obtenida a partir de la lectura de los proyectos artísticos, de tal manera que se facilite la comprensión y el análisis de los mismos. Las fichas RAE contemplan los siguientes aspectos: Número de RAE, autor, correo electrónico, título, año, unidad patrocinante, resumen, abstract, palabras clave, problema de investigación, objetivos, metodología, principales referentes teóricos conceptuales, resultados obtenidos, conclusiones, bibliografía citada y tipo de producto. La muestra correspondió a la totalidad de los trabajos de grado desarrollados durante el periodo 2004-2014.

El diseño de la investigación es el siguiente:

a. Elaboración de listado de proyectos: se inició con la elaboración del listado de los proyectos artísticos que se han sustentado en el programa de Formación Musical, ya que en la base de datos de egresados del programa la información está consignada de manera individual y por año y semestre, en donde se abordan entre otros aspectos, información relativa a nombre de los proyectos sustentados, fecha de sustentación, énfasis cursado.

Para desarrollar el listado se utilizó Excel, incluyendo: año y semestre de sustentación de los trabajos, Nombre de autor, Énfasis cursado, Nombre del trabajo, observaciones, Persona a cargo de leer el proyecto, si el proyecto es aprobado 
para subir a la base de datos y si este ya se subió. Estas dos últimas columnas sirvieron para llevar un control de los proyectos que se iban subiendo a la base de datos. Como criterios para determinar si el trabajo estaba listo para incluirse a la base de datos se tuvieron los siguientes: buena redacción, ortografía, manejo de espacios y contenido.

Posteriormente se hizo una agrupación de los autores que habían desarrollado el mismo trabajo y ya con este listado se procedió a adjudicar los proyectos a cargo de cada uno de los miembros del proyecto de investigación.

b. Adjudicación de los proyectos de lectura: la investigación contó con el acompañamiento de 8 estudiantes de la carrera de Formación Musical, correspondientes a los distintos énfasis, que fueron vinculados como auxiliares de investigación. Teniendo en cuenta que en el listado se detectaron 279 proyectos, estos fueron distribuidos para su lectura y revisión, de acuerdo con el énfasis que los estudiantes cursaban o en los que los 4 docentes investigadores del trabajo imparten sus asignaturas.

c. Lectura de proyectos y elaboración o validación de RAES: luego de hacer la distribución de los proyectos se inició la lectura de los trabajos y la elaboración de las fichas RAE faltantes, o validación y complementación de las existentes. Para esto se dieron orientaciones con relación a los contenidos y escritura de los aspectos que debían incluirse en el RAE y se hizo un seguimiento semanal a nivel grupal e individual con los estudiantes. En estos encuentros se dio espacio para la discusión y la resolución de dudas sobre temáticas relativas a la revisión y/o redacción de los componentes de la ficha RAE.

Para hacer la revisión de las fichas los estudiantes fueron asignados de a dos por cada docente. Los profesores debían revisar semanalmente redacción, contenido y presentación. Después de estar revisada y aprobada esta información por parte del docente asignado, se enviaban las fichas a la docente investigadora principal del proyecto para revisión y aprobación. Los aspectos a describir e interpretar fueron los pertenecientes a los RAE: Título del Proyecto, Año de conclusión del trabajo, Resumen, Palabras clave, Problema de investigación, Objetivos, Metodología, Principales referentes teóricos conceptuales, Resultados obtenidos, Conclusiones y Bibliografía citada.

La lectura de los proyectos del año 2014 se dificultó debido a que estos trabajos aún no estaban disponibles en la biblioteca de la universidad, por lo cual se debió recurrir directamente a sus autores. En el proceso de búsqueda de los proyectos artísticos se trató de contactar con los autores. Finalmente hay 7 proyectos perdidos que no son tenidos en cuenta para el análisis de la información debido al desconocimiento sobre el contenido de los mismos.

d. Elaboración de formato en Google Drive: paralelamente al desarrollo de los RAES se desarrolló un formato en Google Drive con el fin de facilitar el levantamiento de la información para su posterior análisis. En este formato se incluyó toda la información relevante para la investigación: nombre del proyecto, autor, año de finalización, semestre de finalización, énfasis cursado, temática desarrollada, línea de investigación del proyecto, resumen en español y en inglés, palabras clave, si el proyecto cuenta con planteamiento del problema, problema de investigación, pregunta de investigación, objetivo general, objetivos específicos, objetivos, justificación, antecedentes, marco teórico, metodología, tipo de estudio, diseño de la investigación, técnicas de 
recolección de datos, si cuenta con producto adicional al trabajo escrito y en qué consiste el producto, si cuenta con apartado de bibliografía, porcentaje de bibliografía digital, idioma de las referencias, año de las referencias, si se relacionaba a la música colombiana, temática relativa a esta música, región de Colombia que era abordada en el proyecto, coherencia entre planteamiento del problema y objetivos, diseño metodológico y objetivos, planteamiento, diseño y objetivos, persona que diligenció el formulario, nombre de asesor específico, de asesor metodológico y si el proyecto cuenta o no con RAE al inicio de la investigación. Este formato fue revisado y luego de ser aprobado se realizó una reunión grupal con el fin de brindar las directrices para el diligenciamiento de la información y se inició su desarrollo.

e. Revisión y validación de la información de Excel: al finalizar el diligenciamiento del formulario de Google Drive se exportó la base de datos en Excel, se revisó por parte de todo el equipo y se validó la información que allí se encontraba para pasarla a SPSS.

f. Análisis de datos desde SPSS por énfasis: luego de pasar la información a SPSS versión 15, se realizó un análisis de frecuencias y se exportaron las tablas. A partir de esta información se realizó una descripción de los hallazgos al interior de cada uno de los énfasis y se hizo un informe, abarcando todas las categorías de análisis del proyecto. Los aspectos analizados fueron: número de proyectos vinculados a la línea de Investigación-Creación, Tipo de estudio de los proyectos, de diseños de la investigación, técnicas de recolección de datos empleadas y productos de los proyectos artísticos.

g. Elaboración del informe final

\section{Resultados}

\section{Número de proyectos vinculados a la línea de investigación-creación}

Tabla 1. Relación entre las temáticas de los proyectos

con las líneas de investigación de la Facultad de Artes

\begin{tabular}{|c|c|c|c|c|c|c|c|c|c|c|c|c|}
\hline \multirow{3}{*}{ Categoría } & \multicolumn{10}{|c|}{ Énfasis } & \multirow{2}{*}{\multicolumn{2}{|c|}{ Programa }} \\
\hline & \multicolumn{2}{|c|}{ Arreglos } & \multicolumn{2}{|c|}{ Composición } & \multicolumn{2}{|c|}{ Ejecución } & \multicolumn{2}{|c|}{ Enseñanza } & \multicolumn{2}{|c|}{ Ingeniería } & & \\
\hline & Frec & $\%$ & Frec & $\%$ & Frec & $\%$ & Frec & $\%$ & Frec & $\%$ & Frec & $\%$ \\
\hline Investigación-creación & 58 & 86,6 & 14 & 77,8 & 25 & 29,1 & 2 & 6,3 & 21 & 33,3 & 120 & 45,1 \\
\hline Educación y arte & 4 & 6 & 1 & 5,6 & 31 & 36 & 24 & 75 & 5 & 7,9 & 65 & 24,4 \\
\hline $\begin{array}{c}\text { Gestión, desarrollo y } \\
\text { producción }\end{array}$ & 1 & 1,5 & 0 & 0 & 2 & 2,3 & 1 & 3,1 & 12 & 19 & 16 & 6,0 \\
\hline Teoría, estética y arte & 4 & 6 & 1 & 5,6 & 24 & 27,9 & 1 & 3,1 & 4 & 6,3 & 34 & 12,8 \\
\hline $\begin{array}{l}\text { Salud, ciencia, } \\
\text { tecnología y artes }\end{array}$ & 0 & 0 & 0 & 0 & 2 & 2,3 & 4 & 12,5 & 21 & 33,3 & 27 & 10,2 \\
\hline $\begin{array}{c}\text { Subjetividad, desarrollo } \\
\text { y espacios sociales: }\end{array}$ & 0 & 0 & 2 & 11,1 & 2 & 2,3 & 0 & 0 & 0 & 0 & 4 & 1,5 \\
\hline Total & 67 & 100 & 18 & 100 & 86 & 100 & 32 & 100 & 63 & 100 & 266 & 100,0 \\
\hline
\end{tabular}

Frec: Frecuencia (cantidad de proyectos)

En la tabla anterior se puede evidenciar que todos los trabajos tienen relación con las líneas de investigación de la Facultad y que la línea con mayor producción es la 
de Investigación-Creación con 120 proyectos, representando el 45,1\% de los trabajos desarrollados. Asimismo, se puede establecer que el énfasis en Arreglos Musicales es el que tiene el porcentaje mayor de trabajos vinculados a Investigación-Creación, con un 86,6\%. Le sigue Composición con el 77,8\%. Además, Enseñanza Instrumental representa el porcentaje más bajo de trabajos vinculados a esta línea, con el 6,3\%.

\section{Tipo de estudio de los proyectos}

Tabla 2. Tipo de estudios de los proyectos

\begin{tabular}{|c|c|c|c|c|c|c|c|c|c|c|c|c|}
\hline \multirow{3}{*}{ Categoría } & \multicolumn{10}{|c|}{ Énfasis } & \multirow{2}{*}{\multicolumn{2}{|c|}{ Programa }} \\
\hline & \multicolumn{2}{|c|}{ Arreglos } & \multicolumn{2}{|c|}{ Composición } & \multicolumn{2}{|c|}{ Ejecución } & \multicolumn{2}{|c|}{ Enseñanza } & \multicolumn{2}{|c|}{ Ingeniería } & & \\
\hline & Frec & $\%$ & Frec & $\%$ & Frec & $\%$ & Frec & $\%$ & Frec & $\%$ & Frec & $\%$ \\
\hline Análisis Comparativo & 2 & 3 & 1 & 5,6 & 3 & 3,5 & 2 & 6,3 & 3 & 4,8 & 11 & 4,1 \\
\hline $\begin{array}{c}\text { Análisis comparativo y } \\
\text { experimental: }\end{array}$ & 0 & 0 & 0 & 0 & 1 & 1,2 & 0 & 0 & 0 & 0 & 1 & 0,4 \\
\hline $\begin{array}{l}\text { Análisis comparativo y } \\
\text { exploratorio; }\end{array}$ & 1 & 1,5 & 0 & 0 & 0 & 0 & 0 & 0 & 0 & 0 & 1 & 0,4 \\
\hline $\begin{array}{c}\text { Investigación } \\
\text { Documental } \\
\end{array}$ & 4 & 6 & 1 & 5,6 & 2 & 2,3 & 1 & 3,1 & 0 & 0 & 8 & 3,0 \\
\hline Correlacional & 0 & 0 & 0 & 0 & 0 & 0 & 1 & 3,1 & 0 & 0 & 1 & 0,4 \\
\hline Estudio de caso & 0 & 0 & 1 & 5,6 & 0 & 0 & 2 & 6,3 & 1 & 1,6 & 4 & 1,5 \\
\hline $\begin{array}{c}\text { Descriptivo y } \\
\text { exploratorio }\end{array}$ & 3 & 4,5 & 0 & 0 & 2 & 2,3 & 1 & 3,1 & 5 & 7,9 & 11 & 4,1 \\
\hline $\begin{array}{l}\text { Descriptivo y } \\
\text { bibliográfico }\end{array}$ & 0 & 0 & 0 & 0 & 1 & 1,2 & 0 & 0 & 0 & 0 & 1 & 0,4 \\
\hline $\begin{array}{l}\text { Descriptivo y } \\
\text { experimental }\end{array}$ & 2 & 3 & 0 & 0 & 2 & 2,3 & 0 & 0 & 0 & 0 & 4 & 1,5 \\
\hline $\begin{array}{c}\text { Descriptivo-analítico- } \\
\text { exploratorio }\end{array}$ & 1 & 1,5 & 0 & 0 & 0 & 0 & 0 & 0 & 0 & 0 & 1 & 0,4 \\
\hline El proyecto se perdió & 0 & 0 & 0 & 0 & 0 & 0 & 0 & 0 & 0 & 0 & 0 & 0,0 \\
\hline Estudio Analítico & 0 & 0 & 0 & 0 & 2 & 2,3 & 0 & 0 & 1 & 1,6 & 3 & 1,1 \\
\hline Estudio descriptivo & 27 & 40,3 & 3 & 16,7 & 49 & 57 & 8 & 25 & 23 & 36,5 & 110 & 41,4 \\
\hline Estudio experimental & 11 & 16,4 & 2 & 11,1 & 8 & 9,3 & 2 & 6,3 & 17 & 27 & 40 & 15,0 \\
\hline $\begin{array}{c}\text { Experimental y } \\
\text { explicativo }\end{array}$ & 1 & 1,5 & 0 & 0 & 0 & 0 & 0 & 0 & 1 & 1,6 & 2 & 0,8 \\
\hline Exploratorio & 11 & 16,4 & 5 & 27,8 & 14 & 16,3 & 3 & 9,4 & 10 & 15,9 & 43 & 16,2 \\
\hline Investigación-Acción & 0 & 0 & 0 & 0 & 1 & 1,2 & 9 & 28,1 & 2 & 3,2 & 12 & 4,5 \\
\hline Investigación-creación & 2 & 3 & 5 & 27,8 & 1 & 1,2 & 0 & 0 & $\mathrm{o}$ & $\mathrm{o}$ & 8 & 3,0 \\
\hline $\begin{array}{c}\text { Sistematización de } \\
\text { experiencias: }\end{array}$ & 2 & 3 & 0 & 0 & 0 & 0 & 3 & 9,4 & 0 & 0 & 5 & 1,9 \\
\hline Total & 67 & 100 & 18 & 100 & 86 & 100 & 32 & 100 & 63 & 100 & 266 & 100,0 \\
\hline
\end{tabular}

Fuente: tomado de Investigar desde la práctica musical. Los proyectos artísticos en el programa de Formación Musical de la Universidad El Bosque (Morales, et al., 2018, p. 114).

En cuanto a los tipos de estudio, en la Tabla 2 se observa que todos los énfasis presentan Análisis Comparativos, Estudios Descriptivos, Exploratorios y Experimentales. Con relación a los Análisis Comparativos, el porcentaje de elaboración de proyectos utilizando este tipo de estudios es bajo $(4,1 \%)$ y el énfasis que presenta el porcentaje de estudios más alto en esta categoría es el de Enseñanza con un 6,3\%, siendo Arreglos el más bajo, con el 3\%. 
Algunos de los trabajos se desarrollan utilizando dos o tres tipos de estudio, esto sucede en cuanto a Análisis Comparativos con estudios experimentales en el énfasis de Ejecución con el 1,2\% de los trabajos y en Estudios Comparativos y Exploratorios en el énfasis de Arreglos con un 1.5\%. En ambos casos, los valores corresponden a un solo (Morales, et al., 2018, p. 112).

La mayoría de los trabajos corresponden a Estudios Descriptivos $(41,4 \%)$, siendo Ejecución el énfasis con el porcentaje más alto en este tipo de trabajos (57\%), seguido por el de Arreglos con un 40,3\%. El énfasis con el porcentaje más bajo es el de Composición con tres trabajos que representan el 16,7\%.

Hay un total de seis trabajos en el énfasis de Arreglos (9\%) y cinco trabajos en el de Ejecución (5,8\%), para lo casos donde se hacen Estudios Descriptivos agregando otro tipo de estudios. Todos los énfasis, con excepción del énfasis de Composición, presentan trabajos descriptivos combinados con exploratorios.

El énfasis que más presenta Estudios Experimentales es el de Ingeniería, con un porcentaje del $27 \%$ y el que menos trabajos tiene en esta categoría es el de Enseñan$z a$ con un $6,3 \%$.

En todos los énfasis se han desarrollado Estudios Exploratorios, teniendo Composición el porcentaje más alto en esta categoría $(27,8 \%)$, correspondiente a cinco trabajos, y Enseñanza el porcentaje más bajo $(9,4 \%)$ equivalente a tres trabajos.

Todos los énfasis con excepción al de Ingeniería, han desarrollado Investigación Documental. El porcentaje más bajo corresponde a Ejecución con 2,3\% y el más alto es el de Arreglos con el 6\%.

En cuanto a los Estudios de Casos, solo tres énfasis: Composición, Enseñanza e Ingeniería, han realizado trabajos de este tipo, siendo Enseñanza con un 6,3\% la que tiene el porcentaje más alto e Ingeniería con 1,6\% el porcentaje más bajo. (Morales, et al., 2018, p. 116)

Los énfasis que manejan la mayor diversidad de tipos de estudio son los de Enseñanza, con nueve tipos de estudios (Análisis Comparativo, Investigación Documental, Estudio Correlacional, Estudio de Caso, Descriptivo, Experimental, Exploratorio, Investigación-Acción y Sistematización de Experiencias), seguido por el de Ejecución con ocho (Análisis Comparativo, Investigación Documental, Descriptivo, Analítico, Experimental, Exploratorio, Investigación-Acción e Investigación-Creación)

Los demás énfasis presentan cada uno siete tipos de estudios. En Arreglos: Análisis Comparativo, Investigación Documental, Descriptivo, Experimental, Exploratorio, Investigación Creación y Sistematización de Experiencias. En Composición: Análisis Comparativo, Investigación Documental, Estudio de Caso, Descriptivo, experimental, Exploratorio e Investigación-Creación y en Ingeniería: Análisis Comparativo, estudio de caso, Descriptivo, Analítico, Experimental, Exploratorio e Investigación-Acción.

Los estudios que son comunes a todos los énfasis resultaron ser: Análisis Comparativo, Descriptivo, Experimental y Exploratorio.

En cuanto al tipo de estudio que presenta el mayor porcentaje en cada uno de los énfasis, este es el Descriptivo, el cual presenta un porcentaje en los énfasis de Arreglos del 40,3\%, Ejecución con el 57\% e Ingeniería con un 36,5\%. En Enseñanza el tipo de estudios que más se plantea es el de Investigación-Acción con un porcentaje del 28,1\%, mientras que en Composición los porcentajes más altos corresponden a Estudios Exploratorios y de Investigación-Creación, con el 27,8\% en cada uno.

Investigación-Acción se ha desarrollado en trabajos correspondientes a tres énfasis: Ejecución, Enseñanza e Ingeniería. El énfasis con el porcentaje más alto de proyectos de esta categoría es Enseñanza con el 28,1\% y el más bajo es Ejecución con el 1,2\%. 
Solo tres énfasis han especificado en sus proyectos que su metodología se enmarca en Estudios de Investigación-Creación: Arreglos, Composición y Ejecución, teniendo Composición el del porcentaje más alto con un 27,8\% y el de Ejecución el de porcentaje más bajo con el 1,2\%. Solamente los énfasis de Ejecución e Ingeniería han desarrollado Estudios Analíticos, teniendo el porcentaje más alto Ejecución con el 2,3\% y el más bajo el de Ingeniería con el 1,6\%.

Con relación a la Sistematización de Experiencias solo los énfasis de Arreglos y Enseñanza han realizado estudios de este tipo, siendo Enseñanza el de mayor porcentaje con el 9,4\% y Arreglos, el menor, con el 3\%. Solamente el énfasis de Enseñanza ha desarrollado Estudios Correlacionales, correspondiendo a un porcentaje de 3,1\%. (Morales, et al., 2018, p. 112.

\section{Diseños de la investigación}

De acuerdo a la Tabla 3, un 68,4\% de los trabajos presentan el diseño de la investigación, teniendo Composición el mayor porcentaje de trabajos que contemplan esta información con un 94,4\%, seguido por Enseñanza con el 78,1\%, Arreglos con el $70,1 \%$ y Ejecución con $69,8 \%$. El énfasis con el menor porcentaje de trabajos con diseño de investigación es el de Ingeniería con el 52,4\%.

Tabla 3. Proyectos que presentan el diseño de la investigación

\begin{tabular}{|c|c|c|c|c|c|c|c|c|c|c|c|c|}
\hline \multirow{3}{*}{ Categoría } & \multicolumn{10}{|c|}{ Énfasis } & \multirow{2}{*}{\multicolumn{2}{|c|}{ Programa }} \\
\hline & \multicolumn{2}{|c|}{ Arreglos } & \multicolumn{2}{|c|}{ Composición } & \multicolumn{2}{|c|}{ Ejecución } & \multicolumn{2}{|c|}{ Enseñanza } & \multicolumn{2}{|c|}{ Ingeniería } & & \\
\hline & Frec & $\%$ & Frec & $\%$ & Frec & $\%$ & Frec & $\%$ & Frec & $\%$ & Frec & $\%$ \\
\hline No:2 & 20 & 29,9 & 1 & 5,6 & 26 & 30,2 & 7 & 21,9 & 30 & 47,6 & 84 & 31,6 \\
\hline Sí:3 & 47 & 70,1 & 17 & 94,4 & 60 & 69,8 & 25 & 78,1 & 33 & 52,4 & 182 & 68,4 \\
\hline Total & 67 & 100 & 18 & 100 & 86 & 100 & 32 & 100 & 63 & 100 & 266 & 100,0 \\
\hline
\end{tabular}

Cabe resaltar que los diseños constituyen el plan de acción a seguir para garantizar que realmente se va a recoger de manera pertinente toda la información necesaria para alcanzar los objetivos de los proyectos (Morales, et al., 2018, p. 112). Teniendo en cuenta que en el programa de Formación Musical los proyectos se desarrollan a partir de tres tipos macro de diseños: desarrollo de la experiencia y sistematización simultánea, reflexión teórico-artística posterior a la experiencia y reflexión teórico-artística anterior a la experiencia, los cuales contienen los diseños de investigación propios a los tipos de estudio empleados para la fase de indagación o reflexión de las propuestas y que la práctica investigativa se desarrolla a partir de las fases de los diseños de investigación, es interesante ver que en un número considerable de trabajos no se ha hecho una reflexión del procedimiento de los proyectos, o por lo menos se puede deducir que si esta reflexión se llegó a realizar no aparece en la metodología que es donde debería quedar plasmada para la entrega del informe final del trabajo desarrollado.

Los trabajos que no contemplaban el diseño de la investigación fueron analizados para determinar en su desarrollo cuál había sido el diseño empleado. Al analizar el diseño de la totalidad de los trabajos se establecieron tres macro diseños: Bucle, Ortodoxo e Invertido. En la Figura 1 se presenta un gráfico con los tres tipos de diseños. 


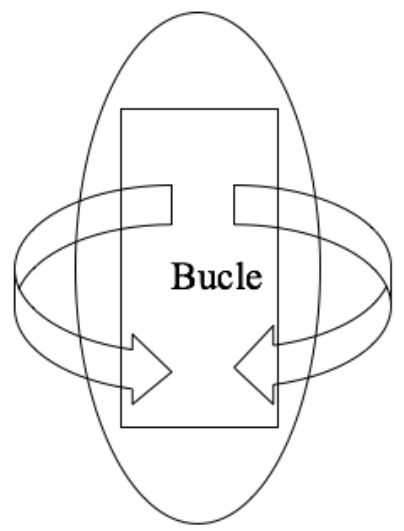

\section{Ortodoxo}

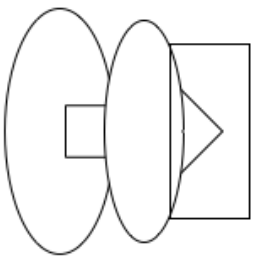

\section{Invertido}

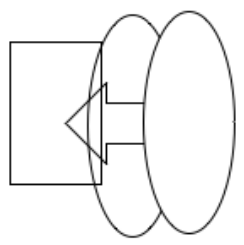

Figura 1. Diseños de los Proyectos Artísticos

Fuente: tomado de Investigar desde la práctica musical. Los proyectos artísticos en el programa de Formación Musical de la Universidad El Bosque (Morales, et al., 2018, p. 106)

Los trabajos de grado en el programa de Formación Musical se desarrollan a partir de tres tipos de diseños: desarrollo de la experiencia y sistematización simultánea $(\mathrm{Bu}-$ cle), reflexión teórico-artística posterior a la experiencia (Invertido) y reflexión teóricoartística anterior a la experiencia (Ortodoxo), los cuales corresponden a las dinámicas de la obra musical que se estudia o se desarrolla en el proyecto. (Morales, et al., 2018, p. 105)

Cabe resaltar que algunos trabajos ejemplarizan los modelos anteriormente mencionados de forma clara, y que otros se prestan para converger las posibilidades etéreas que enmarcan la creación musical desde diferentes ámbitos y propósitos.

Dentro de los trabajos que responden al modelo ortodoxo, se encuentra la realización de la Música para un cortometraje; trabajo realizado por Sebastián Vázquez Sepúlveda, del énfasis en Composición del Programa de Formación Musical de la Universidad El Bosque; trabajo titulado Composición de música original para el cortometraje animado-mudo de 1946, "A Christmas Dream". El proyecto plantea la creación de la música original del cortometraje mediante procesos de investigación que involucran la música para cine y su rigor técnico. La particularidad de este cortometraje es que no tiene sonido, ya que es un filme mudo que se debe reproducir en un proyector casero de $8 \mathrm{~mm}$ marca Bell and Howell.

Uno de los aspectos que determinan su estructuración se evidencian en la metodología, en ella se desarrolla un estudio analítico-descriptivo de corte cualitativo; su diseño es el siguiente:

- Búsqueda de información sobre el contexto histórico, descripción del documental, extracción de técnicas de composición, sesión de spotting, análisis dramático musical. proceso compositivo, elaboración del informe final.

Como ejemplo del modelo Invertido, Reflexión teórico artística posterior a la experiencia, el proyecto desarrollado por Vanesa Rodríguez Mejía, titulado Análisis vocal del Currulao: una comparación entre dos cantoras del Pacífico Sur. Este proyecto consiste en un análisis comparativo de la interpretación vocal del 
Currulao entre dos cantoras del Pacífico Sur. En este se establecen características a partir de un análisis basando en videos y entrevistas a las dos cantadoras. Este trabajo es un análisis comparativo de corte cualitativo. Su diseño es el siguiente:

- Definición del problema, Objetivos Generales y específicos, Desarrollo del marco teórico, Elaboración y validación de las herramientas para la recolección de datos, Recolección de datos, desarrollo de entrevistas y grabaciones, Sistematización de la información, Descripción e interpretación de la información, Yuxtaposición, Comparación, Elaboración de las reflexiones y sugerencias para los cantantes de música popular interesados en aprender a interpretar Currulao, Conclusiones y estructuración del informe final y Como técnicas de recolección de datos se realizan entrevistas y análisis de videos.

Por último, se tienen proyectos que adoptan el modelo de bucle, los proyectos cuyo desarrollo de la experiencia y sistematización se realiza de forma simultánea. Un ejemplo de este modelo es el proyecto desarrollado por Laura Ximena Barrios del énfasis en Ingeniería de sonido y corresponde a un Laboratorio: Espacialización en tiempo real usando el sistema Spatium. Este proyecto consiste en ampliar los conceptos de mezcla en tiempo real desarrollados por Óscar Contreras y aplicarlos en un montaje de arte sonoro Multimedia, en el que se pueda apreciar una experiencia sonora espacializada usando el software Spatium. Como complemento al montaje, se desarrolla un laboratorio sobre espacialización sonora, dirigido a la comunidad de la Facultad de artes, con el fin de compartir el conocimiento adquirido en el desarrollo de este proyecto. La propuesta surge de la necesidad de aportar nuevos elementos al montaje y puesta en escena de la banda "Time To Pay" y de la intención de compartir estos conocimientos a la comunidad artística en general, contribuyendo con nuevos conceptos sobre el manejo del sonido en las distintas puestas en escena.

La metodología del proyecto corresponde a un estudio descriptivo y exploratorio de carácter cualitativo. Su diseño es el siguiente:

- Búsqueda bibliográfica del sonido, fundamentos del diseño sonoro y el manejo de Spatium. Experimentos de laboratorio y de campo, Ensayos con la banda y el software Spatium, Elaboración, validación y aplicación de encuestas y entrevistas, Análisis de resultados de encuestas para desarrollar el contenido del taller, elaboración de un taller dirigido a los estudiantes de la facultad de arte, Desarrollo de material de apoyo para el Laboratorio Realización de Taller: Espacialización en tiempo real. Grabación del video y elaboración del diario de campo, Desarrollo de las encuestas al público oyente del Taller, Desarrollo del montaje, Grabación del video y diario de campo, Desarrollo de las encuestas al público asistente al montaje, Análisis de la información, Edición del video final, Elaboración de Conclusiones y del informe final.

Las técnicas de recolección de datos utilizados en este trabajo son la Entrevista, la Encuesta y el Diario.

\section{Técnicas de recolección de datos empleadas}

La Tabla 4 nos indica que el 76,7\% de los proyectos presentan las técnicas de recolección de datos. El énfasis que tiene el porcentaje más alto de trabajos que con- 
tiene este aspecto es el de Composición con el 88,9\%, seguido por Ingeniería con el $85,7 \%$, Enseñanza con el 75\% y Ejecución con el 72,1\%. El énfasis con menor porcentaje de trabajos que contiene esta categoría es el de Arreglos, con un 71,6\%.

Tabla 4. Proyectos que presentan las técnicas de recolección de datos

\begin{tabular}{|c|c|c|c|c|c|c|c|c|c|c|c|c|}
\hline \multirow{3}{*}{ Categoría } & \multicolumn{10}{|c|}{ Énfasis } & \multirow{2}{*}{\multicolumn{2}{|c|}{ Programa }} \\
\hline & \multicolumn{2}{|c|}{ Arreglos } & \multicolumn{2}{|c|}{ Composición } & \multicolumn{2}{|c|}{ Ejecución } & \multicolumn{2}{|c|}{ Enseñanza } & \multicolumn{2}{|c|}{ Ingeniería } & & \\
\hline & Frec & $\%$ & Frec & $\%$ & Frec & $\%$ & Frec & $\%$ & Frec & $\%$ & Frec & $\%$ \\
\hline No:2 & 19 & 28,4 & 2 & 11,1 & 24 & 27,9 & 8 & 25 & 9 & 14,3 & 62 & 23,3 \\
\hline Sí:3 & 48 & 71,6 & 16 & 88,9 & 62 & 72,1 & 24 & 75 & 54 & 85,7 & 204 & 76,7 \\
\hline Total & 67 & 100 & 18 & 100 & 86 & 100 & 32 & 100 & 63 & 100 & 266 & 100,0 \\
\hline
\end{tabular}

En todos los énfasis se desarrollan cuatro técnicas de recolección de datos: Encuesta, Entrevista, Análisis Musical y Observación. Así mismo en todos los énfasis, con excepción del de Composición, se han elaborado diarios de campo, según se evidencia en la Tabla 5.

Tabla 5. Técnicas de recolección de datos empleadas en los proyectos

\begin{tabular}{|c|c|c|c|c|c|c|c|c|c|c|c|c|}
\hline \multirow{3}{*}{$\begin{array}{l}\text { Técnica de } \\
\text { Recolección }\end{array}$} & \multicolumn{10}{|c|}{ Énfasis } & \multirow{2}{*}{\multicolumn{2}{|c|}{ Programa }} \\
\hline & \multicolumn{2}{|c|}{ Arreglos } & \multicolumn{2}{|c|}{ Composición } & \multicolumn{2}{|c|}{ Ejecución } & \multicolumn{2}{|c|}{ Enseñanza } & \multicolumn{2}{|c|}{ Ingeniería } & & \\
\hline & Frec & $\%$ & Frec & $\%$ & Frec & $\%$ & Frec & $\%$ & Frec & $\%$ & Frec & $\%$ \\
\hline Encuesta & 7 & 4,8 & 1 & 3,6 & 11 & 7,0 & 4 & 6,8 & 18 & 17,0 & 41 & 15,4 \\
\hline Entrevista & 19 & 13,0 & 5 & 17,9 & 33 & 20,9 & 16 & 27,1 & 29 & 27,4 & 102 & 38,3 \\
\hline $\begin{array}{l}\text { Análisis } \\
\text { musical }\end{array}$ & 49 & 33,6 & 12 & 42,9 & 54 & 34,2 & 6 & 10,2 & 5 & 4,7 & 126 & 47,4 \\
\hline Observación & 27 & 18,5 & 3 & 10,7 & 28 & 17,7 & 9 & 15,3 & 13 & 12,3 & 80 & 30,1 \\
\hline $\begin{array}{c}\text { Diario de } \\
\text { campo }\end{array}$ & 22 & 15,1 & 0 & 0,0 & 8 & 5,1 & 11 & 18,6 & 28 & 26,4 & 69 & 25,9 \\
\hline Otras & 22 & 15,1 & 7 & 25,0 & 24 & 15,2 & 13 & 22,0 & 13 & 12,3 & 79 & 15,9 \\
\hline Total & 146 & 100,0 & 28 & 100,0 & 158 & 100,0 & 59 & 100,0 & 106 & 100,0 & 497 & 100,0 \\
\hline
\end{tabular}

Fuente: tomado de Investigar desde la práctica musical. Los proyectos artísticos en el programa de Formación Musical de la Universidad El Bosque (Morales, et al., 2018, p. 120)

El Análisis Musical es la técnica de recolección de datos más utilizada (47,4 \%). El porcentaje más alto donde se utiliza esta técnica es en el énfasis de Arreglos con un 33,6\%, mientras el porcentaje más bajo en la utilización de esta técnica lo tiene Ingeniería con un 4,7\%. (Morales, et al., 2018, p. 118)

El uso de esta técnica es relevante por el objeto de estudio de los proyectos y pone de manifiesto que los estudiantes cuentan con una formación musical que les permite no solo analizar la música, sino también transcribirla y editarla, lo cual es indispensable en el desarrollo profesional de un músico. Cabe resaltar que el uso de estas técnicas es lo que permite que la música de transmisión oral tenga permanencia y reconocimiento.

En cuanto a la Encuesta, esta técnica es empleada con el porcentaje más alto en el énfasis de Ingeniería con el 17\%, seguido por Ejecución con el 7\%. El porcentaje más bajo lo tiene Composición con el 3,6\%. 
La Entrevista se desarrolla con el porcentaje más alto en el énfasis de Ingeniería con un 27,4\%. El porcentaje más bajo lo tiene Arreglos con un $13 \%$.

En cuanto a la Observación, esta técnica es empleada principalmente en el énfasis de Arreglos con un 18,5\% y el énfasis de porcentaje más bajo en esta categoría es el de Composición con un 10,7\%. (Morales, et al., 2018, p. 119)

En cuanto al Diario de Campo, esta técnica se emplea en mayor medida en el énfasis de Ingeniería con un porcentaje del 26,4\%. En el énfasis de Composición no se utiliza esta técnica.

Los énfasis de Arreglos, Composición y Ejecución coinciden en que la técnica de recolección de datos que mayormente utilizan es la del Análisis musical, cuyos porcentajes son los más altos en relación con las demás técnicas de recolección de datos, mientras que para el énfasis de Ingeniería esta es la técnica que menos se utiliza. (Morales, et al., 2018, p. 119)

En cuanto a las técnicas que se emplean menos en cada uno de los énfasis, ya que cuentan con el porcentaje más bajo de uso, son la de la Encuesta en los énfasis de Arreglos y Enseñanza, mientras que el Diario de Campo es la menor empleada en los énfasis de Composición y en Ejecución. En Ingeniería la técnica que menos se emplea es el Análisis Musical. (Morales, et al., 2018, p. 120)

\section{Productos de los proyectos artísticos}

Todos los trabajos presentan, al menos, un producto adicional al documento escrito del proyecto artístico, según se aprecia en la Tabla 6. Como una fortaleza de los productos se destaca la diversidad de los mismos y se evidencia que en su mayoría corresponden a los énfasis cursados, pero también puede verse una transversalidad de los mismos, ya que estos consisten en un $12,0 \%$ en una Propuesta Pedagógica, las cuales se desarrollan especialmente en los énfasis de Enseñanza instrumental, Ejecución e Ingeniería de Sonido. Un 20,0\% son Composiciones y principalmente se dan en los énfasis de Arreglos y Composición, y un 10,8\% corresponde a Materiales Didácticos Audiovisuales, desarrollados mayoritariamente en los trabajos de los énfasis de Enseñanza, Ejecución e Ingeniería de Sonido.

E1 $57 \%$ de los proyectos pertenecen a una categoría diferente a la de las cuatro propuestas iniciales: Propuesta Pedagógica, Composición, Página Web y Material Didáctico Audiovisual, en donde la frecuencia mayor fue la de DVD o Cd de audio y/o video, con 157 trabajos que tenían este tipo de producto. Así mismo solo se presentó un libro, quince estudios, Cartillas, Guías, o manuales, cinco juegos interactivos o videojuegos, una aplicación interactiva y una página Web.

Tabla 6. Tipo de productos de los proyectos

\begin{tabular}{|c|c|c|c|c|c|c|c|c|c|c|c|c|}
\hline \multirow{3}{*}{ Categoría } & \multicolumn{10}{|c|}{ Énfasis } & \multirow{2}{*}{\multicolumn{2}{|c|}{ Programa }} \\
\hline & \multicolumn{2}{|c|}{ Arreglos } & \multicolumn{2}{|c|}{ Composición } & \multicolumn{2}{|c|}{ Ejecución } & \multicolumn{2}{|c|}{ Enseñanza } & \multicolumn{2}{|c|}{ Ingeniería } & & \\
\hline & Frec & $\%$ & Frec & $\%$ & Frec & $\%$ & Frec & $\%$ & Frec & $\%$ & Frec & $\%$ \\
\hline Propuesta pedagógica & 1 & 0,8 & 1 & 6,7 & 19 & 13,1 & 23 & 46 & 7 & 7,78 & 51 & 12,0 \\
\hline Composición & 39 & 31,2 & 14 & 93,3 & 21 & 14,5 & 2 & 4 & 9 & 10 & 85 & 20,0 \\
\hline Página web & 0 & 0 & 0 & 0 & 0 & 0 & 0 & 0 & 1 & 1,11 & 1 & 0,2 \\
\hline Didáctico-audiovisual & 6 & 4,8 & 0 & 0 & 16 & 11 & 5 & 10 & 19 & 21,1 & 46 & 10,8 \\
\hline Otros & 79 & 63,2 & 0 & 0 & 89 & 61,4 & 20 & 40 & 54 & 60 & 242 & 57,0 \\
\hline Total & 125 & 100 & 15 & 100 & 145 & 100 & 50 & 100 & 90 & 100 & 425 & 100,0 \\
\hline
\end{tabular}




\section{Discusión}

Teniendo en cuenta las seis líneas de investigación de la Facultad de Artes de la Universidad El Bosque, se puede apreciar que el porcentaje de trabajos más alto se da en la de Investigación-Creación con 122 trabajos, correspondiente al 43,7\% de la muestra. Cabe resaltar que los trabajos de esta línea están relacionados directamente a los énfasis de Arreglos, Composición y Ejecución, principalmente, y que en estos se realiza una producción musical a partir de una investigación sobre tendencias, estilos o lenguajes musicales.

El porcentaje de trabajos relacionados a la línea de Investigación-creación, denota el empoderamiento del papel de artista-músico por parte del estudiantado vinculado al programa de Formación Musical, ya que este tipo de investigación, tal como señala Daza (2009) "es considerada una manera a través de la cual las artes posicionan sus procesos creativos para estar al nivel de la comunidad académica científica" (p. 87).

La Investigación-Creación en la manera en la que se desarrolla en los proyectos artísticos analizados, evidencia mediante sus procesos y productos, los aprendizajes adquiridos al interior de los énfasis y contribuye al enriquecimiento del hecho sonoro. Este tipo de proceso investigativo tal como lo señala Scanner (2009) "permite manifestar lo que permanece contenido en la expresión misma de la creación artística, como lo es la teoría enlazada y aplicada a una práctica" (p. 234). Estos trabajos enriquecen el ejercicio profesional del egresado ya que como señala este mismo autor en este tipo de trabajos el estudiante "se concentrará en una problemática específica en la que profundizará, y paralelamente realizará una labor de creación y exploración" (Scanner, 2009, p. 324).

Al respecto, se pudo constatar que en las fases de investigación los estudios desarrollan modelos característicos de las ciencias sociales en todos los énfasis ya que todos tienen en común el haber realizado análisis comparativos, estudios descriptivos, exploratorios y experimentales. Asimismo, es de resaltar que la mayor parte de los trabajos corresponden a estudios descriptivos, siendo Ejecución el énfasis que presenta el porcentaje más alto en este tipo de trabajos (57\%), seguido por los énfasis de Arreglos con un 40,3\% y que énfasis con el porcentaje más bajo es el de composición con tres trabajos que representan el 16,7\%.

En cuanto al diseño de los trabajos de Investigación-creación se presentan tres modelos: Desarrollo de la experiencia y sistematización simultánea $(\mathrm{Bu}-$ cle), reflexión teórico-artística posterior a la experiencia (Invertido) y reflexión teórico-artística anterior a la experiencia (Ortodoxo). A partir de estos diseños se evidencia que en los trabajos se desarrolla una fase de investigación, que pueden darse al inicio del proyecto y que a partir de esta se genera un producto artístico resultado del proceso de investigación, pero que también la creación del producto artístico puede darse al mismo tiempo que la fase investigativa o anterior al proceso investigativo.

En cuanto a las técnicas de recolección de datos el análisis musical es la más utilizada, siendo el énfasis de Arreglos Musicales el que más la emplea, en un 33,6\% de sus proyectos, mientras que el de porcentaje más bajo en la utilización de esta técnica es en de Ingeniería de Sonidos con un 4,7\%. El uso de esta técnica es relevante por el objeto de estudio de los proyectos y pone de manifiesto que los estudiantes cuentan con una formación musical que les permite no solo analizar la música, sino también transcribirla y editarla, lo cual es indispensable en el desarrollo profesional de un 
músico. Cabe resaltar que el uso de estas técnicas es lo que permite que la música de transmisión oral tenga permanencia y reconocimiento.

Para López-Cano y San Cristóbal (2014) la Investigación-Creación en artes se diferencia de otros tipos de investigación en cuanto a que presenta diferentes características y procedimientos con relación a la recolección de datos, esta situación es evidenciada en esta investigación, donde se podría afirmar que esto se cumple, ya que el análisis musical es la técnica de recolección de datos más utilizada, aunque también se utilizan las técnicas particulares a las investigaciones en ciencias sociales, tales como la entrevista, la encuesta y el diario de campo. Generalmente en los Proyectos Artísticos se desarrollan reflexiones y análisis en torno a un repertorio, un estudio de caso, la elaboración de guías, las propuestas pedagógicas, o el estudio de situaciones puntuales en torno a la creación y ejecución musical.

Con relación a los productos de los proyectos artísticos, el 70\% de los trabajos presenta un producto adicional al documento escrito. Cabe anotar que como fortaleza de estos, se destaca la diversidad de los mismos y se evidencia que en su mayoría corresponden a los énfasis cursados, pero también puede apreciarse una transversalidad de los mismos, ya que por ejemplo las propuestas pedagógica se desarrollan especialmente en los énfasis de Enseñanza instrumental, Ejecución e Ingeniería de Sonido y de otra parte, los materiales didácticos audiovisuales, son desarrollados mayoritariamente en los trabajos de los énfasis de Enseñanza, Arreglos e Ingeniería de Sonido. Es importante resaltar que los temas de investigación de los proyectos artísticos tienen incidencia en el ciclo completo de la producción musical: creación, arreglo, interpretación, registro o grabación y difusión. La identificación y categorización de los productos de cada uno de los énfasis facilitó el relacionamiento con productos propios de las industrias creativas, lo cual a su vez permitió generar reflexiones y sugerencias encaminadas a la estructuración y mejora de futuros.

\section{Conclusiones}

Este estudio sobre los trabajos de grado desarrollados en el programa de Formación Musical de la Universidad El Bosque, permite concluir que desde el año 2004 los proyectos artísticos han contribuido a la formación investigativa, constituyéndose en su mayoría en trabajos de investigación científica o de Investigación-Creación. Una minoría de trabajos son de creación, mas no de investigación en tanto que no responden a una situación problémica ni a una pregunta de investigación.

Se pudo finiquitar que generalmente en los Proyectos Artísticos se desarrollan reflexiones y análisis en torno a un repertorio, un estudio de caso, la elaboración de guías, propuestas pedagógicas, o el estudio de situaciones puntuales en torno a la creación y ejecución musical.

De otra parte, cabe resalar que a partir de las nuevas modalidades de trabajos de grado se pretende contribuir a que los nuevos productos derivados de los proyectos artísticos sean más acordes con los perfiles profesionales de los cinco énfasis que desarrolla el programa, ya que en los procesos de enseñanza que se abordan para el alcance del perfil del egresado no son importantes solamente los procesos formativos tradicionales de cada énfasis, sino que también es relevante el replanteamiento de dichos procesos en el desarrollo de competencias y habilidades dentro de las lógicas 
de producción en las industrias creativas, para lo cual sería pertinente favorecer el desarrollo de ámbitos interdisciplinares de aprendizaje.

La importancia de establecer vínculos con el entorno o con la realidad que rodea al educando, se evidencia en el desarrollo de los proyectos de grado que han propendido por vincular "esa realidad" al quehacer artístico o musical, lo cual se evidencia en que los objetos de estudio de los diferentes proyectos se encuentran inscritos al medio en cual se desenvuelven los estudiantes, y en el que muy probablemente se desempeñarán profesionamente a futuro.

A partir de este estudio se concluye que la producción vinculada a InvestigaciónCreación derivada de los trabajos de grado en el programa de Formación Musical ha sido significativa durante el periodo $2004-2014$, en donde el $45,1 \%$ de los trabajos emplearon esta metodología. De igual manera y a partir del análisis de los diseños de los trabajos, se pueden definir tres diseños macro para el desarrollo de los trabajos de Investigación-Creación: Desarrollo de la experiencia y sistematización simultánea (Bucle), reflexión teórico-artística posterior a la experiencia (Invertido) y reflexión teórico-artística anterior a la experiencia (Ortodoxo) que pueden constituir un aporte para la definición de los diseños en Investigación-Creación.

El hecho de que los trabajos de grado en la modalidad de Investigación-Creación sea tan alto, aun cuando nunca se tuvo esto como requisito para el planteamiento de los mismos, puede deberse a que para este periodo 2004-2014 existía una línea de investigación con dicho nombre en el grupo de investigación al que se encuentra inscrito el programa de Formación Musical. Cabe resaltar que los trabajos en la modalidad de Investigación-Creación evidencian el empoderamiento que los estudiantes del programa han tenido en cuanto a los conocimientos adquiridos en sus énfasis, y su proyección como artistas creadores, en donde la creación se convierte en un medio de producción del conocimiento.

\section{Referencias bibliográficas}

Aldana, G. M. (2012). La formación investigativa: su pertinencia en pregrado., Revista Virtual Universidad Católica del Norte, 1(35), 367-379.

Asprilla, L. (2013). El proyecto de creación-investigación. La investigación desde las artes. Cali: Bellas Artes.

Barrera, M. F. (2006). Líneas de investigación. (2 ${ }^{a}$ ed.). Caracas, Venezuela: Ediciones Quirón-Fundación.

Barriga, M. (2011). La investigación-creación en los trabajos de pregrado y postgrado en Educación Artística.. Revista El Artista. Bogotá. Colombia.

Cabanzo, F. (2015). Grupo de Expresión, artes y creación. Obtenido de http://scienti. colciencias.gov.co:8080/gruplac/jsp/visualiza/visualizagr.jsp?nro=00000000010685

Casas, F. (2008). Hacia una caracterización de la creación y la producción artística, su reconocimiento y valoración. Cali. Colombia.: Universidad del Valle-Acofartes.

Cerda, H. (2007). Por qué y para qué la investigación formativa. Colombia: IX Congreso Departamental de Educación Física Educación Física y Construcción de Ciudadanía.

Daza, S. L. (2009). Investigación creación: Un acercamiento a la investigación en las artes. Horiz. Pedagógico. 11 (1), Iberoamericana-Institución Universitaria.

Escorcia, O. (2010). Manual para la investigación. Guía para la formulación, desarrollo y divulgacióndeproyectos. ObtenidodeUniversidadNacionaldeColombia.Bogotá.Colombia.: http://www.bdigital.unal.edu.co/7551/1/manualparala\%20investigaci\%C3\%B3n.pdf 
García, J. (2004). Estudios descriptivos. Nure Investigación, nº7, Junio. Recuperado de http:// webpersonal.uma.es/ jmpaez/websci/bloqueiii/docbiii/estudios\%20descriptivos.pdf

Gómez, L. (2011). Área de Investigación del programa de Formación Musical en la Universidad El Bosque. Bogotá, Colombia: Universidad El Bosque.

González, B. (s.f.). Módulo de “investigación en el aula. Investigación en el aula. Universidad Sergio Arboleda. Recuperado de http://190.85.246.40/docentes/investigacion_aula.htm

Hernández, C. (2003). Investigación e Investigación formativa. Nómadas, 18., 183-193.

Hernández, R., Fernández, C., \& Baptista, P. (2010). Metodología de la investigación. México, D.F., México.: Mc. Graw Hill.

Hurtado, J. (2010). Líneas de investigación y gerencia del conocimiento: premisas de la cultura de la investigación. Revista Ciencia, tecnología y sociedad, 83-92.

Lebrún, A. (2014). CONSENSUS. Industrias culturales, creativas y de contenidos. Obtenido de Centro de investigaciones: http://www.unife.edu.pe/centro-investigacion/revista/N19 Vol2/\%20Artu00EDculo\%203.pdf

Miyahira, J. (2009). La investigación formativa y la formación para la investigación en el pregrado. Rev Med Hered [online]. 20 (3), 119-122.

Morales, B. y Giraldo, A. (2018). Investigar desde la práctica musical. Los proyectos artísticos en el programa de Formación Musical de la Universidad El Bosque. Bogotá, Colombia.: Editorial Universidad El Bosque.

Restrepo, B. (2003). Conceptos y Aplicaciones de la Investigación Formativa, y Criterios para Evaluar la Investigación científica en sentido estricto. CNA, 1, 1-19.

Restrepo, B. (2008). Formación investigativa e investigación formativa: Acepciones y operacionalización de estaúltimay Contrastecon la investigación Científica en Sentido Estricto. Obtenido de http:/web.unap.edu.pe/web/sites/default\%20/files/Formacion:investigativa-eInvestigacion-formativa.pdf? $\mathrm{w}=1366 \& \mathrm{~h}=768$

Sandoval, Carlos A. (2002). Investigación cualitativa. Santafé de Bogotá. Colombia: ARFO Editores e Impresores Ltda.

Scanner, D. (2009). Estudis Escènics, quaderns de l'Institut del Teatre. Francia: Editorial (castellano). Broch Àlex.

UNESCO. (s.f.). Comprender las industrias creativas. Las estadísticas como apoyo a las politicas públicas. Articulo. Obtenido de Artículo: http://portal.unesco.org/culture/ es/\%20files/30850/11467401723cultural_stat_es.pdf/cultural_stat_es.pdf

Universidad El Bosque. (2005). Verificación $\bar{d}$ e Estándares de Calidad para Registro Calificado Del Programa de Formación Musical. Bogotá, Colombia: Universidad El Bosque.

Universidad El Bosque. (s.f.). Esto es lo que nos hace El Bosque. Recuperado de http://www. uelbosque.edu.co/

Universidad El Bosque. (2015). Informe de Autoevaluación con fines de Acreditación del Programa de Formación Musical. Bogotá, Colombia. Universidad El Bosque.

Yin, R. K. (1993) Applications of Case Study Research. Washington, D. C.: Cosmos Corporation/ Applied Social Research Methods Series, Volume 34. 23

Yin, R. K. Case Study Research: Design and Methods. (1984) Bervely Hills-California: Sage. 